\title{
Kinetic Modeling at Single-Molecule Resolution Elucidates the Mechanisms of Cellulase Synergy
}

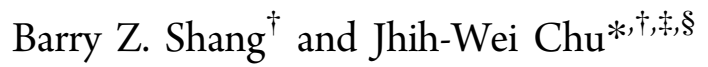 \\ ${ }^{\dagger}$ Department of Chemical and Biomolecular Engineering, University of California, Berkeley, California 94720, United States \\ ${ }^{*}$ Department of Biological Science and Technology, National Chiao Tung University, Hsinchu, Taiwan, ROC \\ ${ }^{\S}$ Institute of Bioinformatics and Systems Biology, National Chiao Tung University, Hsinchu, Taiwan, ROC
}

ABSTRACT: The mechanism of synergistic cooperation between cellulases in decomposing cellulose is revealed by resolving the molecular structures of enzymes and substrates via kinetic modeling. The emergence of endo-exo and exoexo synergy through enzyme-enzyme and enzyme-substrate couplings was investigated with the main exoglucanases (TrCel7A and TrCel6A) and endoglucanase (TrCel7B) of the Trichoderma reesei fungus. The degree of synergy was found to depend on the interplay between two competing effects: (1) enhancement of the complexation rates of exoglucanases with the chain ends created by endoglucanases
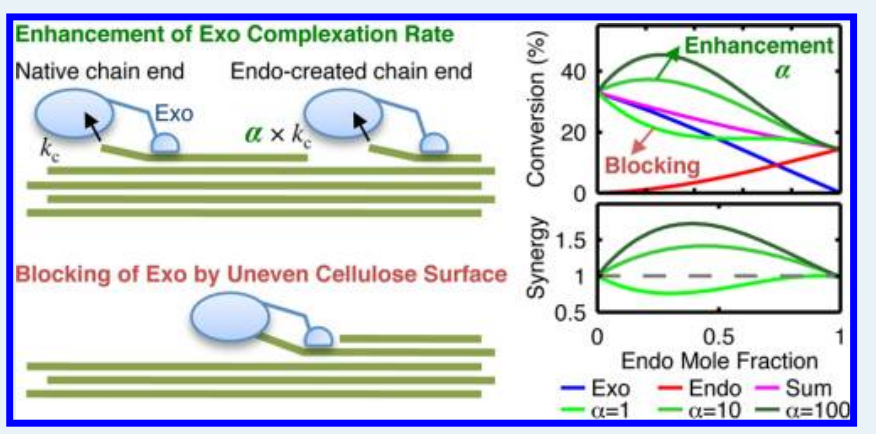

and (2) surface inhibition of processive exoglucanases by

uneven layers of glucan chains on cellulose. We show that the sole effect of TrCel7B in creating more free ends for TrCel7A is insufficient to cause synergistic activity. The combined actions of $\operatorname{TrCel} 7 \mathrm{~A}$ and $\operatorname{TrCel} 7 \mathrm{~B}$ produce a rougher substrate surface that subsequently promotes blocking of processing TrCel7A enzymes. This anti-synergy can be counteracted by enhancing the rates of complexation of TrCel7A with the TrCel7B-created chain ends, allowing their synergistic cooperation. Similarly, kinetic simulations show that exo-exo synergy does not occur if TrCel6A and TrCel7A have only opposite specificities in targeting the two ends of glucan chains. Incorporating endo activity into TrCel6A with complexation rate enhancement, however, can lead to synergy with TrCel7A. Therefore, we find that endo-exo and exo-exo synergies may share the same mechanistic origin. The results of work also highlight that resolving molecular configurations in kinetic modeling allows systematic analysis for elucidating the mechanism of interfacial biocatalysis.

KEYWORDS: enzyme kinetics, interfacial biocatalysis, kinetic modeling, computational biology, cellulase, cellulose

\section{INTRODUCTION}

Cooperation of functionally related proteins in fulfilling complicated tasks is a hallmark of biology. A prominent example is the synergistic increase in the apparent rate of substrate conversion observed upon combination of different cellulases secreted by microorganisms to decompose cellulose in plants. ${ }^{1-6}$ Given a measure of the enzyme activity, such as the substrate conversion $C$ after a certain reaction time, the synergy between two cellulases is defined as $C_{12} /\left(C_{1}+C_{2}\right)$, where $C_{12}$ is the conversion achieved by using cellulase 1 and cellulase 2 together and $C_{1}$ and $C_{2}$ are the conversions achieved by pure cellulase 1 and pure cellulase 2 , respectively, at their corresponding loadings in the mixture. Similar to the origins of many biological processes that rely on interenzyme cooperation, the molecular origins of cellulase synergy are elusive despite its clear functional advantage. Efforts to harness synergy to accelerate cellulose conversion in industrial applications such as biofuel production are thus mostly empirical. ${ }^{7-9}$ In this work, we illustrate that incorporating molecular details of enzymeenzyme and enzyme-substrate couplings into kinetic modeling allows systematic extraction of the mechanistic origins of cellulase synergy. Here, we focus on the cellulases secreted by the fungus Trichoderma reesei, an industry standard with abundant experimental data $^{3,4}$ for developing simulation models.

The main components of the $T$. reesei cellulase cocktail include two exoglucanases (cellobiohydrolases), TrCel7A andTrCel6A, and an endoglucanase, TrCel7B. ${ }^{3,10}$ Exoglucanases generally form complexes with the free ends of glucan chains to perform processive hydrolysis on cellulose, releasing cellobiose into solution after hydrolyzing a glycosidic bond. ${ }^{3,11}$ In conducting processive cleavages, TrCel7A starts from the reducing ends of glucan chains, while TrCel6A initiates the process from the opposing nonreducing ends. ${ }^{1}$ Endoglucanases, on the other hand, can form complexes anywhere within the glucan chain and cleave the chain into shorter fragments. ${ }^{1,3}$ Endoglucanases have limited processivity.

Previous characterizations of the endo-exo synergy between TrCel7A and TrCel7B reveal a few common behaviors. ${ }^{12-22}$ First, the typical synergy values, based on the apparent activity

Received: January 27, 2014

Revised: March 30, 2014

Published: May 20, 2014 


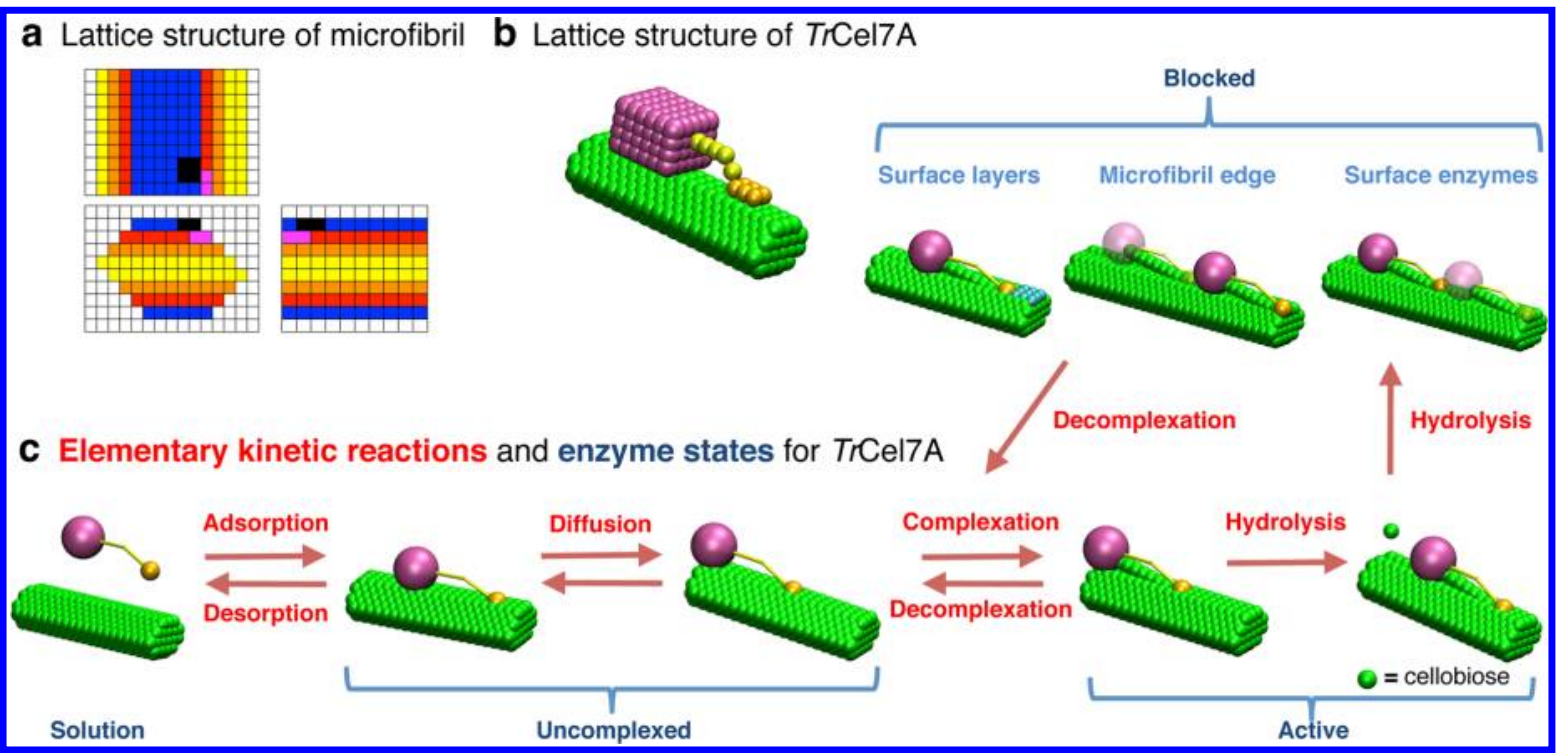

Figure 1. System geometry and elementary kinetic reactions in the stochastic lattice enzyme (SLATE) model. (a) Lattice structure of the cellulose microfibril. The lines indicate the lattice spacing of $5 \AA$. A cellobiose residue is colored black. (b) Lattice representation of a TrCel7A enzyme adsorbed onto a microfibril segment with 20 cellobiose residues per glucan chain. (c) Elementary kinetic steps and enzyme states in the SLATE model for TrCel7A. The kinetic reactions are colored red, and the enzyme states are labeled in blue.

measured by substrate conversion, vary between 1 and $3 .^{3}$ Second, the enzyme composition yielding the highest apparent activity occurs at low endoglucanase mole fractions of $0.1-$ $0.2 .^{12-15}$ Furthermore, the synergy values generally decrease with reaction time, ${ }^{13-19}$ and anti-synergy can be observed at longer times. ${ }^{15,17,20}$ The most common explanation for endoexo synergy is that endoglucanases create more chain ends with which exoglucanases can form complexes. ${ }^{1,6}$ Other theories, based on obstacle removal for TrCel7A by TrCel7B, have also been proposed. ${ }^{19,21,22}$ However, their relevance in causing the aforementioned observations of synergy has not yet been established.

Furthermore, TrCel7A and TrCel6A have been shown to exhibit exo-exo synergy, ${ }^{12,23-25}$ for which the observed values are mostly within the range of $1-3$, as well. ${ }^{3,23}$ Structural characterizations suggest that the semi-open catalytic domains of family 6 cellulases such as TrCel6A likely display endo activity. ${ }^{25,26}$ However, the roles of endo activity in TrCel6A in allowing exo-exo synergy remain unclear. ${ }^{24,27}$

Because cellulase synergy involves collective behaviors of individual enzymes on the substrate surface, it becomes difficult to describe the kinetic mechanisms without resolving the molecular details of interenzyme and enzyme-substrate couplings. $^{4-6}$ Therefore, to reach beyond the classical assumptions of a well-mixed reaction medium and lumped kinetic steps, ${ }^{28-35}$ we developed a spatially resolved model based on the kinetic Monte Carlo (kMC) simulation framework $^{36-38}$ to analyze the roles of spatial configurations in modulating cellulase synergy. In this stochastic lattice enzyme (SLATE) model, the enzyme and glucan chain structures are resolved in three-dimensional space with a $5 \AA$ spatial resolution (Figure 1a,b) to simulate the single-molecule kinetics of all enzymes present in the system. The complexation and decomplexation steps, which are commonly combined as one step in modeling cellulase kinetics, ${ }^{28-31}$ are both explicitly resolved in SLATE. Each cellulase enzyme can perform a full suite of elementary reactions (Figure 1c) consisting of (1) adsorption, (2) desorption, (3) diffusion, (4) complexation, (5) decomplexation, and (6) hydrolysis.

SLATE simulations of TrCel7A alone illustrate that rate limitations of this exoglucanase come mainly from the slow complexation and excessive jamming of the processive enzymes because of obstacles on the substrate surfaces. ${ }^{38}$ Here, for both endo-exo (TrCel7B-TrCel7A) and exo-exo (TrCel6ATrCel7A) mixtures, we resolved interenzyme and enzymesubstrate couplings within SLATE to reveal how synergy emerges from the interplay between slow complexation and interenzyme jamming.

\section{METHODS}

2.1. Stochastic Lattice Enzyme (SLATE) Model. SLATE is a spatially resolved model of the glucan chains of a cellulose microfibril and individual cellulase enzymes that can be used with the kinetic Monte Carlo (kMC) method for studying enzyme kinetics. Reactants in this model are individual enzymes and the cellobiose residues in glucan chains of the cellulose microfibril. Their sizes and shapes are resolved via space-filling cubes with $5 \AA$ sides on a three-dimensional lattice (Figure la,b). The sizes and dimensions of the microfibril substrate are consistent with the crystal structures of these biomolecules. ${ }^{39}$ Each glucan chain is composed of cellobiose residues, and each is modeled as a $1 \mathrm{~nm} \times 0.5 \mathrm{~nm} \times 1 \mathrm{~nm}$ ( or $2 \times 1 \times 2$ lattice units) rectangular prism. The geometry of the microfibril is further described by Chang et $\mathrm{al}^{40}$

The enzyme consists of three structural units: the carbohydrate binding module (CBM), the catalytic domain (CD), and a linker that connects both of them. The CBM is a 2 $\mathrm{nm} \times 1 \mathrm{~nm} \times 3 \mathrm{~nm}$ rectangular prism, while the $\mathrm{CD}$ is a $5 \mathrm{~nm}$ $\times 4 \mathrm{~nm} \times 6 \mathrm{~nm}$ rectangular prism. The dimensions of TrCel7B in the SLATE model are identical to those of TrCel7A. The TrCel6A dimensions are identical to those of TrCel7A, except its $C D$ is a $5 \mathrm{~nm} \times 4 \mathrm{~nm} \times 3 \mathrm{~nm}$ prism to account for its shorter active site tunnel. ${ }^{26,41}$ In our earlier work, ${ }^{38}$ the lattice coordinates for a TrCel7A enzyme adsorbed onto a microfibril segment are provided as an .xyz file to illustrate the enzyme 
structure. For all three enzymes, a $5 \mathrm{~nm}$ linker is employed to connect the two domains. ${ }^{42}$ The enzyme dimensions used in the SLATE model are consistent with their crystal structures. ${ }^{43-47}$

2.2. Elementary Reactions. In the SLATE model, each enzyme in the system is able to perform a set of elementary kinetic reactions: (1) adsorption, (2) desorption, (3) diffusion, (4) complexation, (5) decomplexation, and (6) hydrolysis (Figure 1c) (which are further described in ref 38). Excluded volume constraints are enforced by preventing overlap of the lattice units occupied by reactants.

Adsorption of an enzyme occurs from solution onto the hydrophobic faces of the microfibril surface as described in ref 38. Upon adsorption, the CD is not assumed to be bound to the surface or in a complex with a particular glucan chain. The adsorbed, uncomplexed enzyme is free to diffuse along the surface via its CBM until the enzyme either desorbs or forms a complex with a chain.

The TrCel7A enzyme is allowed to form a complex with only the reducing end of glucan chains, while TrCel6A forms a complex with only the nonreducing end ${ }^{27}$ or additionally with the interior regions of glucan chains if it is provided with the endo activity. ${ }^{25,26}$ For both TrCel7A and TrCel6A, hydrolysis involves cleaving a glycosidic bond, releasing cellobiose into solution, and translation of the enzyme forward along the complexed glucan chain by the length of a cellobiose unit to place the next cellobiose residue at the active site. Both TrCel7A and TrCel6A enzymes remain complexed after hydrolysis because they are processive. ${ }^{1,3,25}$ On the other hand, TrCel7B can form a complex anywhere along a chain. For this enzyme, hydrolysis involves the lumped steps of cleaving a glycosidic bond and decomplexation with the chain, which captures the nonprocessivity of this endoglucanase. Once a cellobiose residue is released into solution, the corresponding lattice units that were filled beforehand are removed from the substrate. The gradual degradation of the microfibril is thus captured, and the dynamic changes of substrate morphology are coupled to enzymatic kinetics in the SLATE model.

2.3. Kinetic Rate Constants. The kinetic rate constant for each elementary kinetic reaction in the SLATE model is listed in Table 1. Literature sources for referencing these rate constants are also listed. In ref 38, these rate constants were shown to yield conversion profiles in agreement with those from experiments. ${ }^{48}$ The consistency of our kinetic parameters with those of other modeling strategies ${ }^{34,49,50}$ was also established. ${ }^{38}$ In this prior work, a key finding from exploring the parameter space of SLATE simulations is that different

Table 1. Kinetic Rate Constants for TrCel7A, TrCel7B, and TrCel6A Used in SLATE Simulations

\begin{tabular}{|c|c|c|c|c|}
\hline rate constant $\left(\mathrm{s}^{-1}\right)$ & $\operatorname{TrCel} 7 A^{a}$ & TrCel7B & TrCel6A & refs \\
\hline adsorption, $k_{\mathrm{a}}$ & $8.9 \times 10^{-4}$ & $8.9 \times 10^{-4}$ & $8.9 \times 10^{-5}$ & $\begin{array}{c}34,53 \\
54\end{array}$ \\
\hline desorption, $k_{\mathrm{h}}$ & $1.0 \times 10^{-3}$ & $1.0 \times 10^{-3}$ & $1.0 \times 10^{-4}$ & $\begin{array}{c}34,53 \\
54\end{array}$ \\
\hline diffusion, $k_{\text {diff }}$ & $1.0 \times 10^{4}$ & $1.0 \times 10^{4}$ & $1.0 \times 10^{4}$ & 61 \\
\hline complexation, $k_{\mathrm{c}}$ & $5.5 \times 10^{-3}$ & $3.0 \times 10^{-3}$ & $5.5 \times 10^{-3}$ & 38,52 \\
\hline $\begin{array}{l}\text { decomplexation, } \\
k_{\mathrm{dc}}\end{array}$ & $1.0 \times 10^{-3}$ & - & $\begin{array}{l}1.0 \times 10^{-3} b \\
10.0^{c}\end{array}$ & 49,50 \\
\hline hydrolysis, $k_{\mathrm{h}}$ & 7.1 & 9.2 & 14 & $\begin{array}{l}51,56 \\
58,59\end{array}$ \\
\hline
\end{tabular}

${ }^{a}$ See ref 38 for details. ${ }^{b}$ For non-endo TrCel6A. ${ }^{c}$ For endo TrCel6A. processes can become rate-limiting in cellulose decomposition depending on the substrate and reaction conditions.

The kinetic rate constants for $\operatorname{TrCel} 7 \mathrm{~A}$ were described in our previous work. ${ }^{38}$ Here, the kinetic rate constants for TrCel7B and TrCel6A are described. The adsorption and desorption rates of $\operatorname{TrCel} 7 \mathrm{~B}$ are assumed to be similar to those of TrCel7A, following the modeling strategy of Levine et al. ${ }^{34}$ The hydrolysis rate of TrCel7B is set to $9.2 \mathrm{~s}^{-1}$. $^{51}$ The complexation rate of $\mathrm{TrCel} 7 \mathrm{~B}$ is adjusted such that the cellulose conversion from $\mathrm{TrCel} 7 \mathrm{~B}$ alone is approximately half the value from TrCel7A alone. This level of lower conversion by pure TrCel7B is generally observed in experiments. ${ }^{12,13,16-19}$ The resulting best-fit value is $3.0 \times 10^{-3} \mathrm{~s}^{-1}$. The slow rate of complexation for $\operatorname{TrCel} 7 \mathrm{~B}$ is also consistent with the results of Maurer et al. ${ }^{52}$ After complexation, the TrCel7B enzyme is only allowed to hydrolyze a bond and then decomplex.

The adsorption and desorption rates of TrCel6A are 10 times lower than those of TrCel7A because the binding of TrCel6A is less reversible.$^{53,54}$ The adsorption equilibrium constant is still that of TrCel7A according to experimental measurements. $^{3,23,55}$ The decomplexation rate of TrCel6A was estimated to be $10.0 \mathrm{~s}^{-1}$. This value is consistent with the more flexible tunnel-forming loops of $\mathrm{TrCel}^{2} \mathrm{~A}^{26}$ that impart processivity lower than that of $\mathrm{TrCel} 7 \mathrm{~A}^{9,56}$ and partial endolike character into the enzyme. ${ }^{25,26}$ The higher frequency of opening expected for these loops would result in a decomplexation rate that is faster than that of $\operatorname{TrCel} 7 \mathrm{~A}$, which possesses more closed loops. ${ }^{57}$ The hydrolysis rate constant of TrCel6A is $14 \mathrm{~s}^{-1} .58,59$ The complexation rate of TrCel6A with free and interior chain ends is assumed to be the same as that of TrCel7A $\left(5.5 \times 10^{-3} \mathrm{~s}^{-1}\right)$. This estimation is considered reasonable because both of these enzymes have active site tunnels, ${ }^{4,45}$ and complexation would involve similar molecular steps of removing a chain from the surface and threading it into the active site.

To model cellulase diffusion, the experimental diffusion coefficient is converted into a diffusion rate using random walk theory. ${ }^{60}$ Given an experimental diffusion coefficient ${ }^{61}$ of $1.0 \times$ $10^{-10} \mathrm{~cm}^{2} \mathrm{~s}^{-1}$ and a hopping length of $1 \mathrm{~nm}$, the corresponding diffusion rate constant is $1.0 \times 10^{4} \mathrm{~s}^{-1}$.

We also assumed that for all enzymes, complexation occurs 10 times more quickly with the initial top and bottom layers of the microfibril. This treatment is used to build in the effective structural heterogeneity of plant cellulose into the simulation model. ${ }^{3,11,38} \mathrm{We}$ found that this representation of the microfibril substrate captures the increasing recalcitrance of the substrate during the reaction ${ }^{3,32,62}$ and provides quantitative agreement with experimentally measured conversion profiles. ${ }^{38}$ However, this treatment did not affect the mechanistic trends discussed in this work. The complexation rate constants in the main text (Table 1) refer to the higher rate constants.

It is important to point out that the complexation rate constant inevitably depends on the substrate crystallinity. In this study, the model attempts to simulate the decomposition of a highly crystalline substrate for which cellulase synergy can be exploited for enhancing conversion rates. Kinetic parameters (Table 1) are thus employed accordingly in the case where complexation is rate-limiting. ${ }^{38}$

2.4. Kinetic Monte Carlo Algorithm for SLATE Simulations. For the spatially resolved SLATE model, a lattice kinetic Monte Carlo (kMC) algorithm based on the nullevent sampling scheme ${ }^{37}$ is employed for simulation. The algorithm proceeds as follows. 
(1) Randomly choose a cellobiose residue or an adsorbed enzyme. Each residue and enzyme can be selected with equal probability.

(2a) If a cellobiose residue is chosen, enumerate a list of all possible adsorption reactions at this site.

(2b) If an adsorbed enzyme is chosen, enumerate a list of all possible reactions available to that enzyme, given its state (e.g., uncomplexed, complexed, or blocked) (Figure 1c). For example, an uncomplexed enzyme can diffuse, complex, or desorb, while a blocked enzyme can only decomplex.

(3) From the enumerated list, select a reaction with a probability proportional to its rate constant.

(4) Perform the selected reaction, and update the system state.

(5) Advance time by the increment $1 /\left(N k_{\max }\right) . N$ is the total number of cellobiose residues adsorbed by enzymes. $k_{\max }$ is a sufficiently large "normalization" constant that defines the time scale of a single null-event kMC iteration. Details about how to choose the parameter are provided in ref 37.

(6) Repeat steps 1-5 until a specified time has elapsed.

Difficulty arises from the high rate of diffusion that introduces stiffness into this numerical scheme, as most $\mathrm{kMC}$ reactions become uninteresting diffusive hops. To overcome this issue, we applied a stochastic quasi-equilibrium technique ${ }^{63}$ for spatial kMC simulations, in which "effective" slow rate constants are determined by averaging over the diffusive jumps. Further details are provided in ref 38 . The result is a $>100$-fold increase in computational speed.

The kMC framework applied here is advantageous compared to other approximate schemes of simulating stochastic processes ${ }^{30,31}$ because the computed kinetic rates constants can be mapped to the phenomenological rates used in massaction models. ${ }^{36}$ The kMC model therefore can be transparently compared with the classical kinetic models, preserves a well-defined physical time scale, and rigorously captures the spatiotemporal evolution defined by the enzyme reaction network.

\section{RESULTS AND DISCUSSION}

3.1. Endo-Exo Synergy Can Be Caused by Complexation Rate Enhancement. The SLATE simulation system consists of a single microfibril of 36 glucan chains with a degree of polymerization of 1024 . The total number of enzyme molecules is set to 18 for an initial surface coverage of $25 \%$ that is within the typical range employed in experimental investigations. ${ }^{12,18,33}$ Kinetic rate constants for elementary reactions (Table 1) are taken from the literature and further detailed in Methods. These parameters yield simulated conversion profiles in agreement with experimental measurements. $^{38}$

We distinguished the rate constant of TrCel7A complexation with a native, preexisting chain end, $k_{\mathrm{c}, 7 \mathrm{~A}}$, from that with a newly created end via an endo cut, $\alpha k_{c, 7 \mathrm{~A}}$ using the enhancement factor $\alpha$. The cases with $\alpha$ values of 1, 10, and 100 were studied. The $\alpha$ values of $>1$ indicate that the complexation rate constant of $\operatorname{TrCel} 7 \mathrm{~A}$ with the endo-created chain ends is enhanced. This enhancement is based on the fact that the active site cleft of TrCel7B is $\sim 50 \AA$ long and would deconstruct a 10-residue glucan chain upon complexation. ${ }^{64,65}$ The glucan chain segment near the endo-created end is thus expected to have looser hydrogen bonding with the other chains on the cellulose surface, and its susceptibility to complexation with $\operatorname{TrCel} 7 \mathrm{~A}$ would hence be higher. The effect of endoglucanases and other cellulases on disrupting the surface structures of cellulose has been inferred from experimental studies $^{66-72}$ but is explicitly linked here to the exo activity via SLATE to assess its impact on synergy.

The conversion versus time profiles (Figure 2) for the cellulose decomposition reactions with pure $\operatorname{TrCel} 7 \mathrm{~A}$, pure

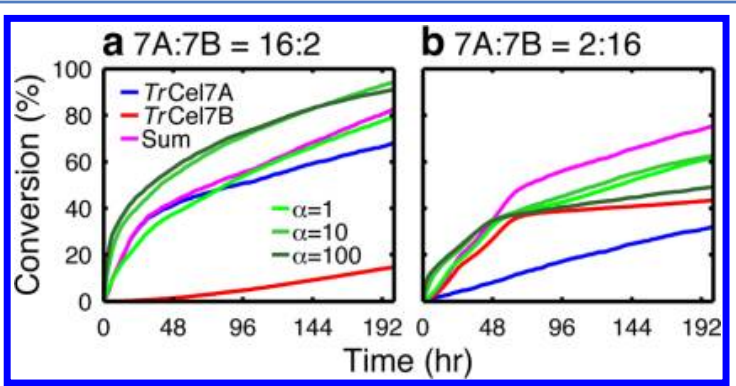

Figure 2. Substrate conversion vs time for the mixtures of TrCel7A and $\operatorname{TrCel} 7 \mathrm{~B}$ at two compositions and three values of complexation enhancement factor $\alpha(1,10$, and 100). The blue lines represent the conversions achieved by TrCel7A enzymes alone. The red lines represent the conversions achieved by using only $\operatorname{TrCel} 7 \mathrm{~B}$ to decompose the cellulose microfibril. The magenta lines represent the sum of the conversions of pure TrCel7A and TrCel7B at their loading levels in the mixture and are the null-synergy conversions. The green lines indicate the actual mixture conversions for different values of $\alpha$. The mixture composition is given as the number of TrCel7A to the number of TrCel7B, where "number" is the actual number of enzyme molecules in the simulation: (a) 16:2 and (b) 2:16.

TrCel7B, and their mixtures illustrate the importance of $\alpha$ in modulating the synergy value. To reveal the general trends, the conversion profiles at two extreme enzyme ratios in the mixture are illustrated. In each conversion profile, the sum of the pure enzyme conversions is also plotted to represent the case of synergy equaling 1 . This null-synergy scenario corresponds to the theoretical conversion when the two types of enzymes act independently on the surface.

In the absence of complexation rate enhancement $(\alpha=1)$, the enzyme mixture exhibits no synergy (Figure 2 ). At the lower end of the TrCel7B mole fraction, the mixture conversion is very close to the null-synergy value (Figure $2 \mathrm{a}$ ). At the higher end of the TrCel7B mole fraction, the mixture conversion is anti-synergistic $(<1)$ (Figure $2 \mathrm{~b}$ ). Therefore, if the action of $\mathrm{TrCel} 7 \mathrm{~B}$ were only to increase the number of reducing ends without affecting the rate of TrCel7A complexation $(\alpha=1)$, endo-exo synergy would not appear in SLATE simulations. A lack of synergy is also observed over intermediate enzyme concentrations when $\alpha=1$ (results not shown). In the presence of complexation rate enhancement $(\alpha$ $=10$ and 100), synergy can be achieved at shorter times $(<24$ h) for both mixture compositions (Figure 2) and is maintained at longer times when the $\mathrm{TrCel} 7 \mathrm{~B}$ mole fraction is low (Figure 2a). However, at higher TrCel7B mole fractions, anti-synergy gradually develops with time $(>24 \mathrm{~h})$ (Figure $2 \mathrm{~b})$. Under these conditions, the $\alpha=10$ conversion approaches the $\alpha=1$ conversion at later times, while the $\alpha=100$ conversion actually falls below. Therefore, the initial increase in activity from the complexation rate enhancement of TrCel7A is lost because of anti-synergistic interactions.

The substrate conversions over the initial $(12 \mathrm{~h})$, medium $(24 \mathrm{~h})$, and final $(72 \mathrm{~h})$ stages of reaction and the corresponding synergy values were analyzed as a function of enzyme concentration (Figure 3). When $\alpha>1$, the calculated 


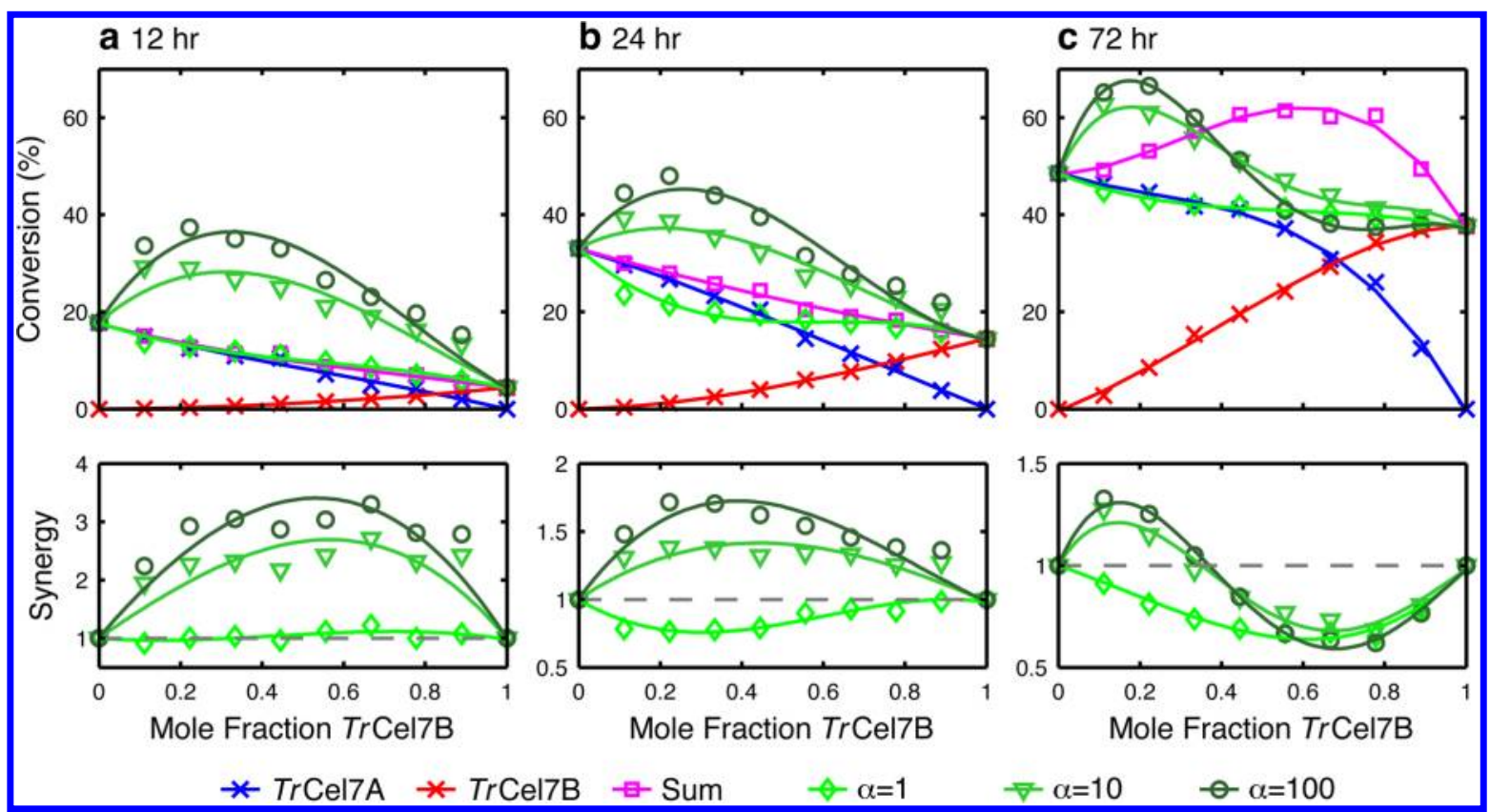

Figure 3. Substrate conversions and synergy values vs TrCel7B mole fraction for the enzyme mixture with TrCel7A for different values of $\alpha$ and reaction times. The blue lines with crosses represent the conversion achieved by TrCel7A enzymes alone. The red lines with crosses represent the conversion achieved by only using $\operatorname{TrCel7B}$ to decompose the cellulose microfibril. The magenta lines with squares represent the sum of conversions achieved by pure TrCel7A and TrCel7B enzymes and are the null-synergy conversions. The green lines indicate the mixture conversions and the synergy values at different values of $\alpha$ : (diamonds) $\alpha=1$, (inverted triangles) $\alpha=10$, and (circles) $\alpha=100$. The value of synergy is defined as the mixture conversion divided by the sum of the pure enzyme conversion. The reaction times are (a) 12, (b) 24 , and (c) $72 \mathrm{~h}$.

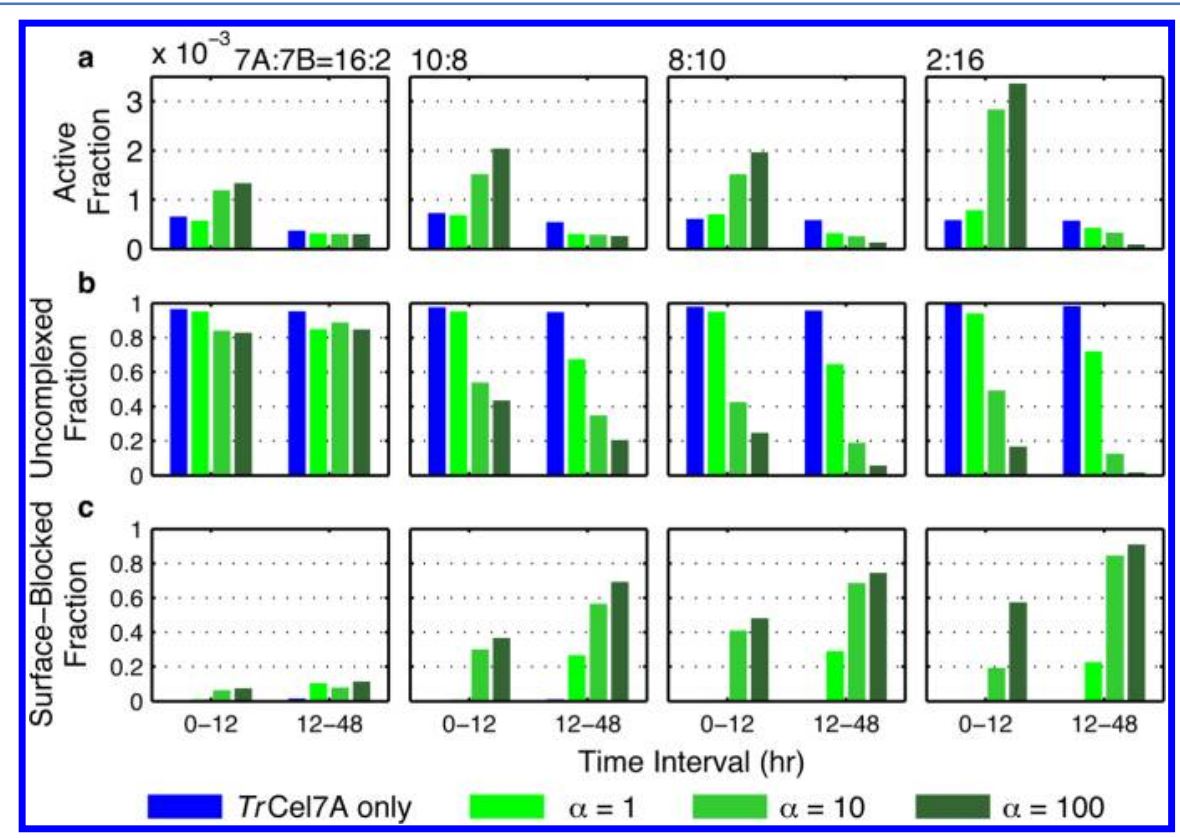

Figure 4. Averaged fractions of TrCel7A enzymes occupied in different states in their pure (blue) and mixture (green) environments at different $\alpha$ values. Averaging was performed over the two intervals of $0-12$ and $12-48 \mathrm{~h}$. The fractions in states are shown for different pure enzyme loadings and mixture compositions represented as the number of TrCel7A to the number of TrCel7B, where "number" is the actual number of enzyme molecules in the simulation. In the pure TrCel7A simulations, the number of TrCel7A enzymes is equal to the number used in the corresponding mixture. The enzyme states illustrated here are the (a) active, (b) uncomplexed, and (c) surface-blocked states.

synergy values are in the range of $1-3$, in accordance with kinetic measurements. ${ }^{3}$ The optimal endoglucanase mole fractions yielding the highest conversions are low $(\sim 0.2)$, and the synergy values also decrease with reaction time (Figure 3), indicating the consistency of SLATE simulations with the experimentally observed trends. ${ }^{12-19}$ To elucidate the molec- ular origins of these synergistic responses, an analysis of singleenzyme kinetics afforded by SLATE modeling is discussed next.

3.2. Single-Enzyme Kinetics Reveals Competing Forces on Synergy. Analysis of the distribution of TrCel7A enzymes over inactive and active states during the reaction allows identification of the kinetic driving forces responsible for synergy. By comparing the time-averaged fractions of TrCel7A 


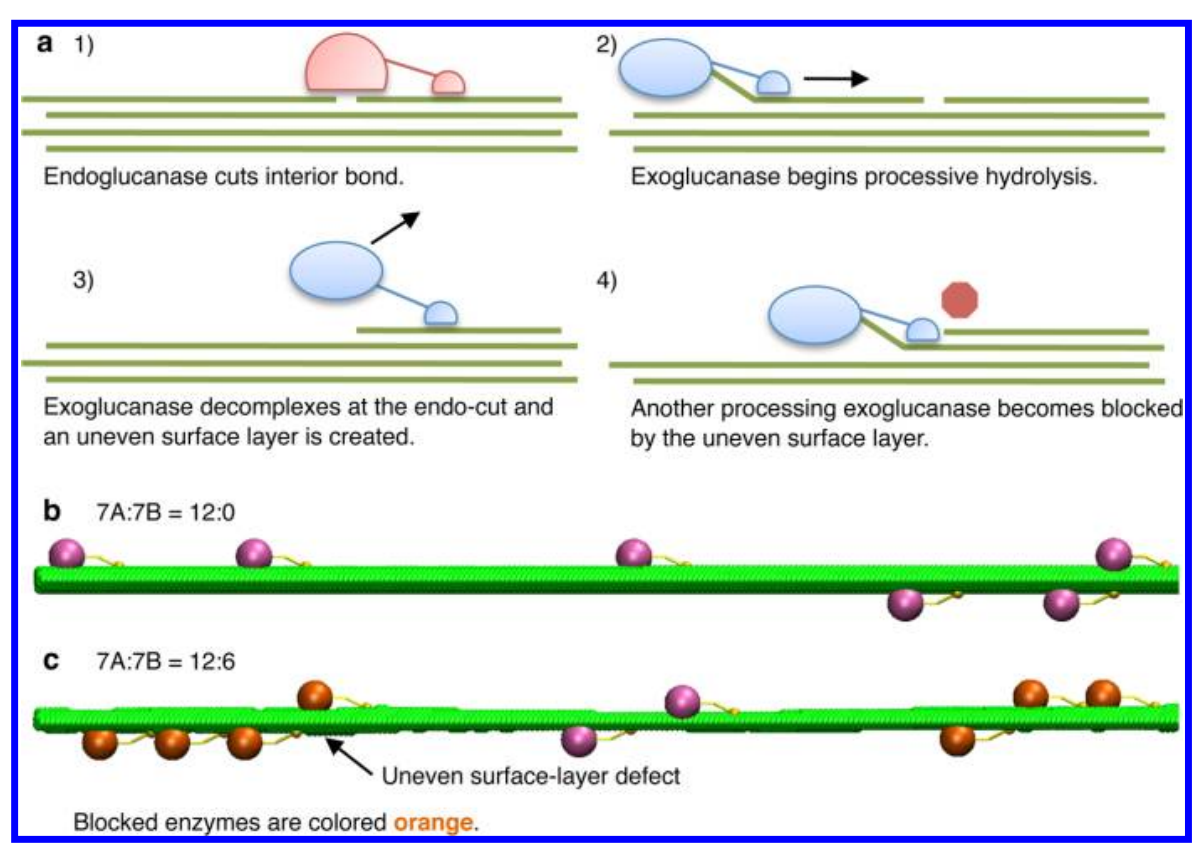

Figure 5. Illustrations of the surface-roughening phenomenon caused by the combined actions of TrCel7A and TrCel7B. (a) The combined actions of $\operatorname{TrCel} 7 \mathrm{~A}$ and $\mathrm{TrCel} 7 \mathrm{~B}$ create a rougher substrate surface that promotes blocking of processing TrCel7A enzymes. Each green line represents a glucan chain. Side views of the microfibril and four layers are shown. (b and c) Snapshots in SLATE simulations illustrating the increase of surface roughness and surface blocking of $\operatorname{TrCel7A}$ enzymes caused by TrCel7B. Only half of the microfibril substrate is shown here for clarity; the reducing end is located on the left-hand side. In both panels b and c, the snapshot is taken after $24 \mathrm{~h}$ of reaction time. (b) Snapshot from a simulation with only 12 TrCel7A enzymes. (c) Snapshot from a simulation with 12 TrCel7A enzymes and 6 TrCel7B enzymes. For comparison to panel b, only the TrCel7A enzymes on the microfibril are shown in panel $c$. The blocked TrCel7A enzymes are colored orange. In panel $c$, a larger fraction of the microfibril has been converted, because of the complexation enhancement provided by $\operatorname{TrCel} 7 \mathrm{~B}$ to $\operatorname{TrCel} 7 \mathrm{~A}$, but the surface is rougher. As a result, surface inhibition of TrCel7A enzymes occurs more frequently to slow the conversion as the reaction proceeds further. A larger fraction of TrCel7A enzymes are therefore blocked as illustrated in panel $\mathrm{c}$.

occupied in different states in the presence and absence of TrCel7B, we elucidated the impact of TrCel7B on TrCel7A activity. For a TrCel7A enzyme that conducts processive cleavages along a glucan chain after complexation, it can be found in one of the following states in the SLATE simulation: (1) solution, (2) uncomplexed, (3) blocked by uneven microfibril surface layers (or surface-blocked), (4) blocked by the microfibril edge, (5) blocked by other adjacent enzymes, or (6) active. An active enzyme is complexed and unblocked by any obstacles (Figure 1c).

The time-averaged fractions of TrCel7A are computed for the uncomplexed, surface-blocked, and active states (Figure 4). The fractions are also separated into two groups as the average from 0 to $12 \mathrm{~h}$ and that from 12 to $48 \mathrm{~h}$. The two time intervals are used here to distinguish the "biphasic" behavior of conversion observed earlier (Figure 2). In the first interval, the burst in the apparent conversion rate can appear in cases when $\alpha>1$. In the second interval, the conversion rate and synergy slow with time. In addition to the active state, we focused on the surface-blocked state of single-molecule kinetics because its fraction dominates over those of the other two blocked states.

In the first time interval, the active fraction of $\operatorname{TrCel} 7 \mathrm{~A}$ in the presence of TrCel7B is greater than that of pure TrCel7A when $\alpha>1$ (Figure 4a). This gain in activity due to complexation rate enhancement corresponds to the initial bursts in substrate conversion (Figure 2). In addition, the fractions of TrCel7A in the uncomplexed state are only significantly lowered in the presence of TrCel7B when $\alpha>1$ (Figure 4b). Without complexation enhancement $(\alpha=1)$, however, the uncomplexed and active fractions in the first interval are relatively unaffected by the addition of TrCel7B (Figure 4b). As discussed next, the fractions of surface-blocked states (Figure 4c) reveal the mechanism of anti-synergy in the mixtures of $\operatorname{TrCel} 7 \mathrm{~A}$ and TrCel7B.

3.3. Surface Inhibition of TrCel7A Can Cause AntiSynergy. The increased fractions of $\mathrm{TrCel} 7 \mathrm{~A}$ in the surfaceblocked state with TrCel7B mole fractions (Figure 4c) reveal anti-synergistic interactions between the two cellulases. SLATE simulations show that the combined actions of endo- and exoglucanases cause more pronounced roughening of cellulose surfaces as the decomposition reaction proceeds (Figure 5a). Consequently, the surface-blocked fraction of TrCel7A is elevated by the presence of $\operatorname{TrCel} 7 \mathrm{~B}$ (Figure $5 \mathrm{~b}, \mathrm{c}$ ). Trajectory analysis of SLATE simulations indicates that after glucan chains are made shorter by the interior endo cuts, a processing TrCel7A molecule is more likely to decomplex before reaching the end of the microfibril. Each decomplexation event in the interior regions then leaves a step defect on the microfibril surface (Figure 5a). This rougher surface presents more obstacles at which the processive motions of TrCel7A enzymes become blocked (Figure 5b,c). Therefore, the surface inhibition of TrCel7A becomes more severe, and the active fraction of TrCel7A decreases as the reaction proceeds (Figure $4 a, c)$. The simulation snapshots illustrate the increased surface blocking of TrCel7A on a roughened microfibril substrate (Figure 5c). The surface blocking is also promoted further at higher mole fractions of TrCel7B and values of $\alpha$ (Figure 4c), as these factors accelerate the formation of obstacles.

At low TrCel7B concentrations, the surface-blocked fractions of $\operatorname{TrCel7A}$ remain at relatively low values even when $\alpha=100$ (Figure 4c), as the development of surface roughening is 
restricted by the smaller number of endo-created ends. In this case, the decrease in the active fraction of TrCel7A over time is less drastic (Figure 4a), and the endo-exo synergy in the substrate conversion can be maintained at longer times (Figure 3c). An optimal balance between complexation enhancement and surface blocking in cellulase synergy is thus found in SLATE simulations to occur at low endoglucanase mole fractions $(<0.2)$ and provides a mechanistic basis for the corresponding experimental observations. ${ }^{12-15}$ The increasing surface roughness with time during cellulose decomposition is also the molecular mechanism identified via SLATE simulations for explaining the decrease in synergy with time observed experimentally. ${ }^{13-19}$ This trend had been hypothesized to be due to loss of reactive sites for endoglucanases over time as the originally buried glucan chains are expected to be more recalcitrant. ${ }^{14,15}$ SLATE simulations indicate that this variation in substrate reactivity is not required for this outcome to occur. These results illustrate that incorporating molecular details in kinetic modeling allows systematic analysis of the effects of different molecular processes on the apparent activity of substrate conversion.

3.4. Endo-Exo Synergy is a Function of Exo Processivity. Given the importance of surface inhibition in affecting synergy as resolved by SLATE simulations, it can be inferred that reduced processivity of TrCel7A could enhance synergy. This prediction is based on the fact that a less processive enzyme has a higher rate of decomplexation ${ }^{38}$ and would be less likely to stay blocked at uneven surface layers. To test this hypothesis, we performed SLATE simulations of TrCel7A with a high decomplexation rate constant of $1.0 \times$ $10^{-1} \mathrm{~s}^{-1}$ and a low decomplexation rate constant of $1.0 \times 10^{-5}$ $\mathrm{s}^{-1}$ while keeping the other rate constants unchanged. For the less processive enzyme, synergy is indeed increased and higher $72 \mathrm{~h}$ conversions above $50 \%$ can be achieved for a broader range of TrCel7B mole fractions. Therefore, having lower exoglucanase processivity builds in higher resistance to surface blocking (Figure 6a), despite the lower intrinsic activity of the individual enzyme molecules. On the other hand, a highly processive enzyme is sensitive to the presence of blocking surface obstacles and exhibits anti-synergy with the addition of TrCel7B (Figure 6b). Prior experiments have pointed to slow decomplexation as a significant rate limitation for TrCel7A alone. ${ }^{49,50}$ Our simulations illustrate that decomplexation can also be a kinetic bottleneck in TrCel7A-TrCel7B mixtures. Maximizing synergy likely requires fine-tuning of both the complexation and decomplexation kinetics.

3.5. Exo-Exo Synergy Does Not Appear without the Endo Activity of TrCel6A. SLATE simulations also reveal that having endo activity in $\operatorname{TrCel} 6 \mathrm{~A}^{25,26}$ is crucial for the exoexo synergy with TrCel7A to emerge. Synergy with TrCel7A was investigated with the SLATE model by using a purely exo TrCel6A and a TrCel6A enzyme having partial endo activity in kinetic simulations. The rate constants employed for both versions of TrCel6A enzymes are listed in Table 1 . The nonendo TrCel6A is allowed to form a complex only with the nonreducing end of glucan chains, while the endo TrCel6A enzyme is able to form a complex with chain interior regions at the same rate as with chain ends. The decomplexation rate constant of endo TrCel6A is also assumed to adopt a higher value (Table 1) because the flexible tunnel-forming loops of TrCel6A are expected to occasionally open to form a cleftlike topology that resembles those of endoglucanases. ${ }^{25,26}$

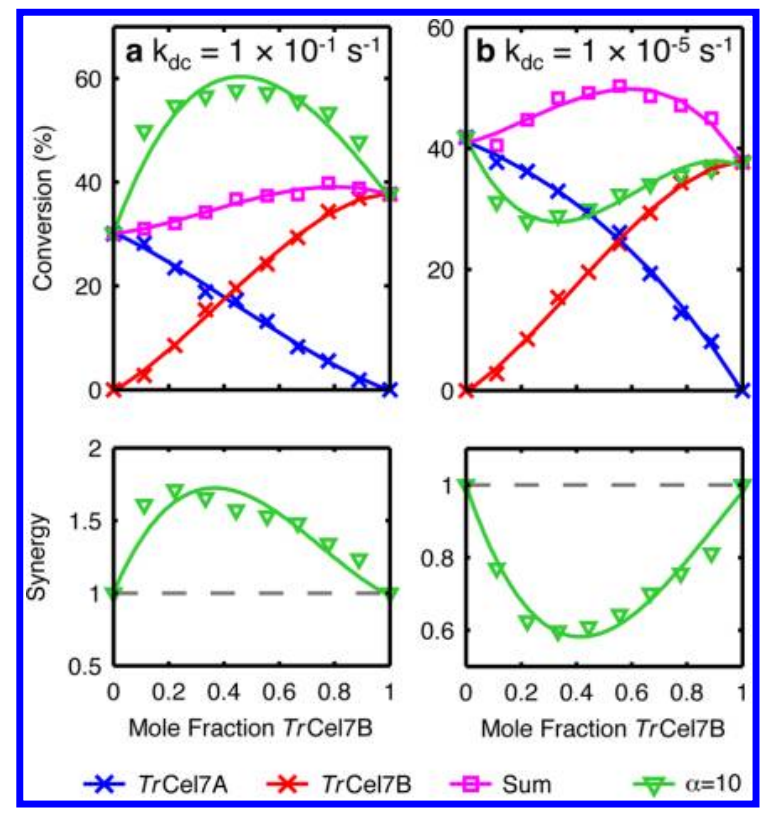

Figure 6. Seventy-two hour substrate conversion and synergy values vs the TrCel7B mole fraction for the enzyme mixture with "mutated" TrCel7A enzymes. The mutation is represented by changing the decomplexation rate of TrCel7A to (a) $1.0 \times 10^{-1}$ or (b) $1.0 \times 10^{-5}$ $\mathrm{s}^{-1}$. The wild-type value is $1.0 \times 10^{-3} \mathrm{~s}^{-1}$. The blue lines with crosses represent the conversions achieved by TrCel7A enzymes alone. The red lines with crosses represent the conversion from using only TrCel7B enzymes to decompose the cellulose microfibril. The magenta lines with squares represent the sum of conversions achieved by pure $\operatorname{TrCel} 7 \mathrm{~A}$ and $\operatorname{TrCel} 7 \mathrm{~B}$ enzymes and are the null-synergy conversions. The green lines with inverted triangles represent the mixture conversions and synergy values with $\alpha=10$. The synergy value is the mixture conversion divided by the sum of pure enzyme conversions.

We found that the synergy values remained relatively low near 1.2 when the endo activity of TrCel6A was absent (Figure $7 a)$ and dramatically increased to $\sim 2.0$ when the endo activity was incorporated (Figure 7b). The latter value is also in accordance with the experimentally observed trends. ${ }^{3,23}$ In these simulations, endo TrCel6A also enhances the complexation rate constant of $\operatorname{TrCel} 7 \mathrm{~A}$ with the newly created chain ends formed by endo cuts by a factor of $\alpha=0$. Therefore, the endo activity and complexation rate enhancement of TrCel6A are indispensible ingredients for the exo-exo synergy to emerge in SLATE simulations. Therefore, it is not sufficient for the two exoglucanases to only have opposite specificities toward chain ends to achieve the experimentally observed values of exo-exo synergy. The involvement of endo activity in the synergy between TrCel7A and TrCel6A had been anticipated previously, ${ }^{25}$ and the SLATE model is employed here to design different simulations to establish the causality and illustrate that exo-exo synergy likely has the same mechanistic origin as endo-exo synergy. In both cases, one enzyme type facilitates the other to overcome the kinetic bottleneck of complexation. ${ }^{38}$

3.6. Discussion. We demonstrated in previous work ${ }^{38}$ that the rate limitations of TrCel7A on cellulose consisted of slow complexation and excessive jamming. On crystalline substrates, we showed that at earlier times, complexation was rate-limiting, but at later times, decomplexation (or dissociation) of enzymes to escape a blocked configuration can become the bottleneck. As a result, maximal conversion achieved by $\operatorname{TrCel} 7 \mathrm{~A}$ is reached by balancing the benefit of having fast complexation with the 


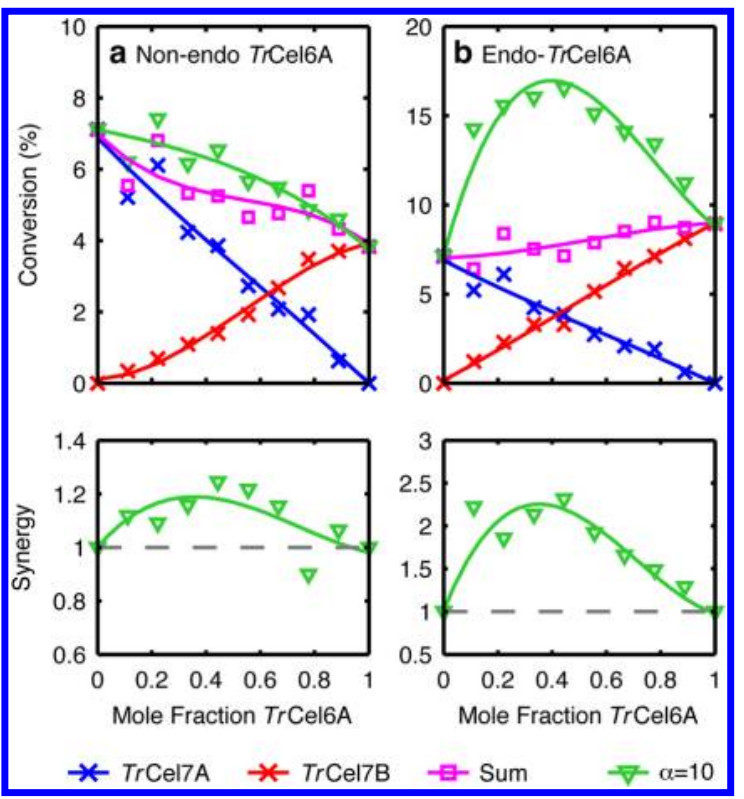

Figure 7. Six-hour substrate conversion and synergy values vs the TrCel6A mole fraction for an enzyme mixture with TrCel7A. Two types of TrCel6A enzymes are studied. (a) The non-endo TrCel6A lacks endo activity and can form a complex only with the nonreducing chain ends. (b) The endo TrCel6A can form a complex with interior chain regions at the same rate as with the nonreducing chain ends and has a higher decomplexation rate constant (Table 1) to reflect its more open active site. The blue lines with crosses represent the conversions achieved by TrCel7A enzymes alone. The red lines with crosses represent the conversion from using only TrCel6A enzymes to decompose the cellulose microfibril. The magenta lines with squares represent the sum of the conversions of pure TrCel7A and TrCel6A enzymes and are the null-synergy conversions. The green lines with inverted triangles represent the mixture conversions and synergy values at $\alpha=10$. The synergy value is the mixture conversion divided by the sum of pure enzyme conversions.

counter effect of enzyme jamming. ${ }^{38}$ Here, we illustrated that the mechanism of balancing complexation and enzyme jamming kinetics is also crucial for different cellulases to exhibit synergy in cellulose decomposition.

A spatially resolved kinetic model, SLATE, is employed to unravel the mechanisms of cellulase synergy at the molecular level. The degree of synergy is found to depend on the interplay between the complexation rate enhancement of exoglucanases and their increased propensity to become blocked by surface obstacles in the presence of endoglucanases. With regard to endo-exo synergy between TrCel7A and TrCel7B, SLATE simulations illustrate that the sole effect of creating more free ends of glucan chains by endoglucanases is insufficient to cause synergy, in contrast to conventional theories. ${ }^{1,6}$ Rather, a necessary requirement for synergy to emerge is complexation enhancement of TrCel7A with endocreated chain ends. We found that an increase in the specific complexation rate constant, rather than free chain end concentration, is the main driver for synergistic conversion. Our findings support the conclusion that endoglucanases are "amorphogenic" ${ }^{\text {"66-72 }}$ or surface-disrupting, but we show that this behavior is required only at the single-chain level to render endo-exo synergy.

By explicitly modeling spatial constraints on the surfaceconfined system, we also found a surface-roughening mechanism that produces anti-synergistic conversion in endo-exo mixtures. We showed that the cellulose surface becomes rougher when exo- and endoglucanases act together on the substrate than the surface treated with TrCel7A alone. In a SLATE simulation, the surface is said to be rough if the density of step configurations such as those shown in Figure 5 is high. The rougher surface promotes the blocking of the processive exoglucanases and thereby reduces their apparent activity. Compared to an endo-free system, a higher concentration of chain defects is formed on the surface, which induces a larger number of processing exoglucanases to become blocked. We found that increasing the decomplexation rate of these enzymes, which enhances their rate of escape from surface inhibition, can promote higher levels of synergistic conversion.

In our SLATE modeling of endo-exo cellulase synergy, TrCel7A and TrCel7B exhibit only exo and endo activity, respectively. This treatment addresses the question of how synergy would arise from combining the two distinct functionalities. As discussed, our results indicate that synergy occurs as a result of balancing complexation enhancement and surface inhibition effects. For cellulases observed in Nature, however, an enzyme can demonstrate both activities with a specific ratio of the two depending on the substrate. For example, Kurašin and Väljamäe showed that TrCel7A may in fact exhibit endo activity. ${ }^{49}$ In this case, TrCel7A molecules would affect each other during cellulose decomposition via complexation enhancement and surface inhibition according to our simulation-derived theory. The apparent activity should thus depend on the interplay between the two effects. This prediction is consistent with the conclusions of Kurašin and Väljamäe ${ }^{49}$ that the length of an obstacle-free path limits the processivity of TrCel7A and that the lumped rate constant of decomplexation plus desorption is an important criterion for selecting cellulases for decomposing cellulose. Therefore, the SLATE model serves as a useful platform for integrating and interpreting experimental data.

In exo-exo mixtures, synergy is also found to depend on the balance between complexation enhancement and surface inhibition. For the mixture of TrCel6A and TrCel7A, SLATE simulations show that synergy does not occur solely from having the two exoglucanases with opposite specificities in complexing with chain ends. Instead, exo-exo synergy emerges when one of the exoglucanases exhibits endo activity and enhances the complexation rate of the other enzyme. As discussed earlier, TrCel7A may also exhibit a certain level of endo activity. ${ }^{49}$ It is thus expected that the endo activity of exoglucanases is likely prevalent, and the resulting complexation enhancement is key for achieving synergy. On the other hand, the decomplexation rate also becomes important for escaping from the more pronounced jammed configurations promoted by the endo activity. SLATE simulations thus offer a molecular mechanism for the observation that dissociation from the complexed chain is an important factor for affecting the apparent activity of cellulose decomposition. ${ }^{49}$ If endo activity were imposed on TrCel7A, we expect that the synergy between TrCel6A and TrCel7A would be enhanced and that the decomplexation rate would need to play a more important role to alleviate surface inhibition effects. In this work, we opted to use purely exo-acting TrCel7A enzymes according to a large number of experimental findings. ${ }^{1,3,4}$ Incorporation of endo activity into $\operatorname{TrCe} 17 \mathrm{~A}^{49}$ in SLATE, though, is straightforward. 


\section{CONCLUSIONS}

The kinetic mechanisms illustrated in this work highlight the importance of spatial resolution at the single-molecule level in describing interfacial biocatalysis. Using a spatially resolved molecular kinetic model, we demonstrate that the dynamic interplay among cellulose enzymes and substrate configurations gives rise to phenomena such as complexation enhancement, surface inhibition, and jamming of enzymes. These phenonmena are found to be key ingredients that determine the degree of kinetic synergy among cellulases and, ultimately, the rate of substrate conversion. In general, further understanding of cellulase synergy and other processes in interfacial biocatalysis likely requires careful consideration of the impact of spatial constraints on reaction mechanisms.

\section{AUTHOR INFORMATION}

\section{Corresponding Author}

*E-mail: jwchu@nctu.edu.tw.

Notes

The authors declare no competing financial interest.

\section{ACKNOWLEDGMENTS}

This work was supported by the Energy Biosciences Institute (Grant OO0J04). The support from the National Chiao Tung University and the Ministry of Science and Technology of Taiwan, Republic of China, via Grant 102-2113-M-009-022MY2 is also acknowledged. We are thankful for the computational resources provided by NERSC (National Energy Research Scientific Computing Center), which is supported by the Office of Science of the U.S. Department of Energy under Contract DE-AC02-05CH11231.

\section{REFERENCES}

(1) Teeri, T. T. Trends Biotechnol. 1997, 15, 160-167.

(2) Wilson, D. B. Curr. Opin. Biotechnol. 2009, 20, 295-299.

(3) Zhang, Y.-H. P.; Lynd, L. R. Biotechnol. Bioeng. 2004, 88, 797824.

(4) Yang, B.; Dai, Z.; Ding, S.-Y.; Wyman, C. E. Biofuels 2011, 2, 421-450.

(5) Van Dyk, J. S.; Pletschke, B. I. Biotechnol. Adv. 2012, 30, 14581480 .

(6) Kostylev, M.; Wilson, D. Biofuels 2013, 3, 61-70.

(7) Nieves, R. A.; Ehrman, C. I.; Adney, W. S.; Elander, R. T.; Himmel, M. E. World J. Microbiol. Biotechnol. 1998, 14, 301-304.

(8) Merino, S. T.; Cherry, J. Adv. Biochem. Eng./Biotechnol. 2007, 108, 95-120.

(9) Lantz, S. E.; Goedegebuur, F.; Hommes, R.; Kaper, T.; Kelemen, B. R.; Mitchinson, C.; Wallace, L.; Stålhberg, J.; Larenas, E. A. Biotechnol. Biofuels 2010, 3, 20.

(10) Nidetzky, B.; Claeyssens, M. Biotechnol. Bioeng. 1994, 44, 961966.

(11) Chundewat, S. P. S.; Beckham, G. T.; Himmel, M. E.; Dale, B. E. Annu. Rev. Chem. Biomol. Eng. 2011, 2, 121-145.

(12) Henrissat, B.; Driguez, H.; Viet, C.; Schülein, M. Nat. Biotechnol. 1985, 3, 722-726.

(13) Karlsson, J.; Medve, J.; Tjerneld, F. Appl. Biochem. Biotechnol. 1999, 82, 243-258.

(14) Boisset, C.; Pétrequin, C.; Chanzy, H.; Henrissat, B.; Schülein, M. Biotechnol. Bioeng. 2001, 72, 339-345.

(15) Jeoh, T.; Wilson, D. B.; Walker, L. P. Biotechnol. Prog. 2006, 22, 270-277.

(16) Medve, J.; Karlsson, J.; Lee, D.; Tjerneld, F. Biotechnol. Bioeng. 1998, 59, 621-634.

(17) Srisodsuk, M.; Kleman-Leyer, K.; Keränen, S.; Kirk, T. K.; Teeri, T. T. Eur. J. Biochem. 1998, 251, 885-892.
(18) Väljamäe, P.; Sild, V.; Pettersson, G.; Johansson, G. Eur. J. Biochem. 1999, 266, 327-334.

(19) Eriksson, T.; Karlsson, J.; Tjerneld, F. Appl. Biochem. Biotechnol. 2002, 101, 41-60.

(20) Andersen, N.; Johansen, K. S.; Michelsen, M.; Stenby, E. H.; Krogh, K. B. R. M.; Olsson, L. Enzyme Microb. Technol. 2008, 42, 362370.

(21) Fox, J. M.; Levine, S. E.; Clark, D. S.; Blanch, H. W. Biochemistry 2012, 51, 442-452.

(22) Jalak, J.; Kurašin, M.; Teugjas, H.; Väljamäe, P. J. Biol. Chem. 2012, 287, 28802-28815.

(23) Medve, J.; Ståhlberg, J.; Tjerneld, F. Biotechnol. Bioeng. 1994, 44, 1064-1073.

(24) Väljamäe, P.; Sild, V.; Petterson, G.; Johannsson, G. Eur. J. Biochem. 1998, 253, 469-475.

(25) Boisset, C.; Fraschini, C.; Schülein, M.; Henrissat, B.; Chanzy, H. Appl. Environ. Microbiol. 2000, 66, 1444-1452.

(26) Zou, J.-y.; Kleywegt, G. J.; Stålhberg, J.; Driguez, H.; Nerinckx, W.; Claeyssens, M.; Koivula, A.; Teeri, T. T.; Jones, T. A. Structure 1999, 7, 1035-1045.

(27) Barr, B. K.; Hsieh, Y.-L.; Ganem, B.; Wilson, D. B. Biochemistry 1996, 35, 586-592.

(28) Converse, A. O.; Optekar, J. D. Biotechnol. Bioeng. 1993, 42, $145-148$.

(29) Zhang, Y.-H. P.; Lynd, L. R. Biotechnol. Bioeng. 2006, 94, 888898.

(30) Asztalos, A.; Daniels, M.; Sethi, A.; Shen, T.; Langan, P.; Redondo, A.; Gnarakan, S. Biotechnol. Biofuels 2012, 5, 55.

(31) Kumar, D.; Murthy, G. S. Biotechnol. Biofuels 2013, 6, 63.

(32) Bansal, P.; Hall, M.; Realff, M. J.; Lee, J. H.; Bommarius, A. S. Biotechnol. Adv. 2009, 27, 833-848.

(33) Levine, S. E.; Fox, J. M.; Blanch, H. W.; Clark, D. S. Biotechnol. Bioeng. 2010, 107, 37-51.

(34) Levine, S. E.; Fox, J. M.; Blanch, H. W.; Clark, D. S. Biotechnol. Bioeng. 2011, 108, 2561-2570.

(35) Berry, H. Biophys. J. 2002, 83, 1891-1901.

(36) Gillespie, D. T. Annu. Rev. Phys. Chem. 2007, 58, 35-55.

(37) Chatterjee, A.; Vlachos, D. G. J. Comput.-Aided Mater. Des. 2007, 14, 253-308.

(38) Shang, B. Z.; Chang, R.; Chu, J. W. J. Biol. Chem. 2013, 288, 29081-29089.

(39) Ding, S.-Y.; Himmel, M. E. J. Agric. Food Chem. 2006, 54, 597606.

(40) Chang, R.; Gross, A. S.; Chu, J. W. J. Phys. Chem. B 2012, 116, $8074-8083$

(41) Varrot, A.; Frandsen, T. P.; Driguez, H.; Davies, G. J. Acta Crystallogr. 2002, D58, 2201-2204.

(42) Beckham, G. T.; Bomble, Y. J.; Matthews, J. F.; Taylor, C. B.; Resch, M. G.; Yarbrough, J. M.; Decker, S. R.; Bu, L.; Zhao, X.; McCabe, C.; Wohlert, J.; Bergenstråhle, M.; Brady, J. W.; Adney, W. S.; Himmel, M. E.; Crowley, M. F. Biophys. J. 2010, 99, 3773-3781.

(43) Kraulis, P. J.; Clore, G. M.; Nilges, M.; Jones, T. A.; Pettersson, G.; Knowles, J.; Gronenborn, A. M. Biochemistry 1989, 28, 72417257.

(44) Rouvinen, J.; Bergfors, T.; Teeri, T.; Knowles, J. K. C.; Jones, T. A. Science 1990, 249, 380-386.

(45) Divne, C.; Ståhlberg, J.; Reinikainen, T.; Ruohonen, L.; Pettersson, G.; Knowles, J. K.; Teeri, T. T.; Jones, T. A. Science 1994, 265, 524-528.

(46) Kleywegt, G. J.; Zou, J. Y.; Divne, C.; Davies, G. J.; Sinning, I.; Stålhberg, J.; Reinikainen, T.; Srisodsuk, M.; Teeri, T. T.; Jones, T. A. J. Mol. Biol. 1997, 272, 383-397.

(47) Mattinen, M.-L.; Linder, M.; Drakenberg, T.; Annila, A. Eur. J. Biochem. 1998, 256, 279-286.

(48) Gusakov, A.; Salanovich, T. N.; Antonov, A. I.; Ustinov, B. B.; Okunev, O. N.; Burlingame, R.; Emalfarb, M.; Baez, M.; Sinitsyn, A. P. Biotechnol. Bioeng. 2007, 97, 1028-1038.

(49) Kurašin, M.; Väljamäe, P. J. Biol. Chem. 2011, 286, 169-177. 
(50) Praestgaard, E.; Elmerdalh, J.; Murphy, L.; Nymand, S.; McFarland, K. C.; Borch, K.; Westh, P. FEBS J. 2011, 278, 15471560.

(51) Claeyssens, M.; van Tilbeurgh, H.; Tomme, P.; Wood, T. M.; McCrae, S. I. Biochem. J. 1989, 261, 819-825.

(52) Maurer, S. A.; Bedbrook, C. N.; Radke, C. J. J. Colloid Interface Sci. 2013, 51, 11389-11400.

(53) Carrard, G.; Linder, M. Eur. J. Biochem. 1999, 262, 637-643.

(54) Palonen, H.; Tenkanen, M.; Linder, M. Appl. Environ. Microbiol. 1999, 65, 5229-5233.

(55) Tomme, P.; Heriban, V.; Claeyssens, M. Biotechnol. Lett. 1980, $12,525-530$.

(56) Igarashi, K.; Uchihashi, T.; Koivula, A.; Wada, M.; Kimura, S.; Okamoto, T.; Penttilä, M.; Ando, T.; Samejima, M. Science 2001, 333, 1279-1282.

(57) Divne, C.; Ståhlberg, J.; Teeri, T. T.; Jones, T. A. J. Mol. Biol. 1998, 275, 309-325.

(58) Harjunpää, V.; Teleman, A.; Koivula, A.; Ruohonen, L.; Teeri, T. T.; Teleman, O.; Drakenberg, T. Eur. J. Biochem. 1996, 240, 584591.

(59) Koivula, A.; Ruohonen, L.; Wohlfahrt, G.; Reinikainen, T.; Teeri, T. T.; Piens, K.; Claeyssens, M.; Weber, M.; Vasella, A.; Becker, D.; Sinnot, M. L.; Zou, J. Y.; Kleywegt, G. J.; Szardenings, M.; Stålhberg, J.; Jones, T. A. J. Am. Chem. Soc. 2002, 124, 10015-10024.

(60) Smit, B.; Maesen, T. L. M. Chem. Rev. 2008, 108, 4125-4184.

(61) Jervis, E. J.; Haynes, C. A.; Kilburn, D. G. J. Biol. Chem. 1997, 272, 24016-24023.

(62) Hong, J.; Ye, X.; Zhang, Y.-H. Langmuir 2007, 23, 1253512540.

(63) Cao, Y.; Gillespie, D. T.; Petzold, L. R. J. Chem. Phys. 2005, 122, 014116.

(64) Lin, Y.; Silvestre-Ryan, J.; Himmel, M. E.; Crowley, M. F.; Beckham, G. T.; Chu, J. W. J. Am. Chem. Soc. 2011, 133, 1661216624.

(65) Lin, Y.; Beckham, G. T.; Himmel, M. E.; Crowley, M. F.; Chu, J. W. J. Phys. Chem. B 2013, 117, 10750-10758.

(66) Gilkes, N. R.; Kilburn, D. G.; Miller, R. C., Jr.; Warren, R. A.; Sugiyama, J.; Chanzy, H.; Henrissat, B. Int. J. Biol. Macromol. 1993, 15, 347-351.

(67) Din, N.; Damude, H. G.; Gilkes, N. R.; Miller, R. C., Jr.; Warren, R. A.; Kilburn, D. G. Proc. Natl. Acad. Sci. U.S.A. 1994, 91, 1138311387.

(68) Gao, P. J.; Chen, G. J.; Wang, T. H.; Zhang, Y. S.; Liu, J. Shengwu Huaxue Yu Shengwu Wuli Xuebao 2001, 33, 13-18.

(69) Joseffson, P.; Henriksson, G.; Wågberg, L. Biomacromolecules 2008, 9, 249-254.

(70) Arantes, V.; Saddler, J. N. Biotechnol. Biofuels 2010, 3, 4.

(71) Cheng, G.; Liu, Z.; Murton, J. K.; Jablin, M.; Dubey, M.; Majewski, J.; Halbert, C.; Browning, J.; Ankner, J.; Akgun, B.; Wang, C.; Esker, A. R.; Sale, K. L.; Simmons, B. A.; Kent, M. S. Biomacromolecules 2011, 12, 2216-2224.

(72) Wang, J.; Quirk, A.; Lipowski, J.; Dutcher, J. R.; Hill, C.; Mark, A.; Clarke, A. J. Langmuir 2012, 28, 9664-9672. 